\title{
El enfoque sistémico en el institucionalismo histórico
}

\section{The systemic approach in historical institutionalism}

\section{Noé Hernández Cortez (iD}

El Colegio de Veracruz

noe.colver@gmail.com

\section{Resumen}

Una de las corrientes representativas en la ciencia política contemporánea es el institucionalismo histórico. En el presente artículo se parte de la premisa de que actualmente predominan dos paradigmas epistemológicos en la ciencia politica: el paradigma de la mecánica clásica y el enfoque sistémico. Se argumenta que el institucionalismo histórico se apoya en la idea de causalidad del paradigma de la mecánica clásica, pero lo ha enriquecido al introducir el enfoque sistémico en el estudio de los procesos politicos. Se concluye que las categorías analíticas como estrategias, trayectorias y coyunturas, propias del institucionalismo histórico, se explican como producto de una visión sistémica del mundo de la politica.

Palabras Clave: Enfoque sistémico, mecánica clásica, procesos políticos, mecanismos causales, ciencia política.

\begin{abstract}
One representative currents in contemporary political science is historical institutionalism. In this article, are based on the premise that currently two epistemological paradigms predominate in political science: the paradigm of classical mechanics and the systemic approach. It is argued that the historical institutionalism is supported by the idea of causality of the paradigm of classical mechanics but has been enriched by introducing the systemic approach in the study of political processes. It is conclude, that analytical categories such as strategies, trajectories and joints, typical of the historical institutionalism, are explained as a product of a systemic vision of the world of politics.
\end{abstract}

Key Words: Systemic approach, classical mechanics, political processes, causal mechanisms, political science.

Articulo: Recibido el 29 de junio de 2018 y aprobado el 7 de noviembre de 2018.

\section{Cómo citar este articulo}

Hernández Cortez, N. (2019). El enfoque sistémico en el institucionalismo histórico. Reflexión Política 21 (41), pp. 134-145. DOI: https://doi.org/10.29375/01240781.3233 


\section{Introducción}

Durante el proceso histórico de la modernidad gradualmente se fragmentó la unidad del conocimiento de la antigua filosofia clásica grecorromana. La ciencia moderna dio origen a la especialización, lo que desembocó en la aparición de distintas ramas delimitadas del conocimiento. La física newtoniana, paradigma de la ciencia moderna, con su nuevo lenguaje científico influyó en la naciente sociología de Augusto Comte en el siglo XIX. En las ciencias naturales, la teoría de la evolución de Charles Darwin fue un paso decisivo para el nacimiento de la ciencia de la biología. Asimismo, la teoría de la evolución influyó en el pensamiento económico de Carlos Marx. A finales del siglo XIX, Herbert Spencer concibió su filosofía social con base en las ideas de la teoría de la evolución de Charles Darwin (Weinstein, 2012). A pesar de la independencia y autonomía adquirida por el conocimiento especializado, no se rompió el diálogo entre las ciencias naturales y las ciencias sociales. Desde una perspectiva epistemológica, podemos decir que existe una larga tradición en las ciencias sociales consistente en mantener un diálogo permanente y fructífero con las ciencias naturales.

La conexión entre las ciencias naturales y las ciencias sociales fue posible a otra novedad de la modernidad: el nacimiento del pensamiento sistémico. La idea de sistema es una idea moderna. De acuerdo con Bunge (1999), el enfoque sistémico nace con la modernidad misma. Así, la noción moderna de sistema permitió pensar la complejidad del mundo fáctico, pues propició la investigación científica de sistemas complejos. Bunge (1999) destaca el enfoque sistémico de la ciencia moderna, por ejemplo, la concepción dinámica del sistema cardiovascular elaborado por William Harvey en los albores del siglo XVII. El enfoque sistémico apareció a la par que las grandes aportaciones científicas del mundo moderno. De esta forma, las nuevas concepciones del mundo natural se expresan en el lenguaje de los sistemas: sistema solar, "sistemas digestivo, nervioso, endocrino, inmune y otros subsistemas del cuerpo humano" (Bunge, 1999, p.8). Si bien es cierto que la modernidad fragmentó el conocimiento, también lo es que la especialización hizo posible que algunos estudiosos establecieran la comunicación entre los distintos campos con base en el enfoque sistémico (Bunge, 2008, 2007a, 2007b, 1999).

El propósito de este artículo es identificar el enfoque sistémico del institucionalismo histórico a través de lo que denominamos el diálogo entre la historia y la ciencia política contemporánea. Este diálogo es producto de la visión sistémica sobre el mundo sociopolítico. Desde la antigüedad grecorromana, la historia jugó un papel importante para explicar fenómenos como el poder y la guerra. Así, con base en el análisis de la historia, el historiador griego Tucídides (1889) obtiene como lección de la Guerra del Peloponeso la siguiente reflexión:

A todos nos conviene la tranquilidad; usar de nuestros bienes en sosiego, y gozar de la paz sin daño ni peligro de nuestras honras y dignidades, y de los otros bienes que se pueden nombrar y contar en largo razonamiento en lugar de los males que, por el contrario, podríamos tener con la guerra. (p. 330)

Podemos afirmar que en esta reflexión del historiador Tucídides prefigura el modelo político de Hobbes (2005) sobre la preferencia estratégica por la paz para el desarrollo material del Estado: el paso razonable de un estado de guerra a un estado de paz a través del contrato político.

Otro ejemplo ilustrativo, sobre las relaciones entre la historia y la ciencia política, es el método de clasificación que aplicó Nicolás Maquiavelo (1971), para identificar los distintos tipos de regímenes políticos con base en el estudio de la historia. Con esta forma de proceder, Maquiavelo contribuye a la metodología de la política comparada contemporánea. Estas conexiones teóricas y metodológicas abonan a la visión sistémica, caracterizada por establecer relaciones entre las distintas disciplinas, en particular entre el estudio de la historia y la ciencia política.

No obstante, la ciencia política contemporánea -altamente especializada- se ha apartado del enfoque sistémico. Sin lugar a duda, que los logros de la ciencia política contemporánea son extraordinarios y esto se 
debe en parte a su concepción mecánica del mundo de la política. Inspirada en los modelos económicos, la ciencia política de la teoría de la elección racional ha simplificado el análisis de la política (Schmitter, 2009). La ciencia política de modelos formales se construyó a partir de la tradición intelectual de Kenneth Arrow, Duncan Black, Allan Gibbard, Mark Sattherwaite, William H. Riker, Anthony Downs, Amartya Sen, Kenneth Shepsle, entre otros (Colomer, 2007). Dentro de la misma ciencia política, la corriente de pensamiento del institucionalismo histórico ha traído de vuelta la complejidad de los sistemas sociales en contextos dinámicos. Para decirlo en el lenguaje de Schmitter (2009), la ciencia política se enfoca en: "la interdependencia de la complejidad del universo político contemporáneo" ${ }^{\prime 1}$ (p. 33).

En este contexto, para conocer las confluencias entre el estudio de la historia y la ciencia política, producto del diálogo entre ambas disciplinas, se plantean las siguientes preguntas de investigación: ¿cuáles son las conexiones epistemológicas entre la historia y la ciencia política? y ¿cuál es el papel analítico que juegan categorías como estrategias, trayectorias y coyunturas en los estudios de procesos históricos que actualmente usa el enfoque sistémico en el institucionalismo histórico? Estas dos preguntas de investigación se intentan contestar en los tres apartados en que se divide el presente trabajo. En el primer apartado se analizan los dos paradigmas epistemológicos que están presentes en la ciencia política contemporánea. En un segundo apartado se expone la relevancia del institucionalismo histórico en la ciencia política, como corriente de pensamiento que ha formulado una teoría coherente que define las categorias de estrategias, trayectorias y coyunturas en la explicación de los procesos políticos. Por último, se presentan conclusiones.

\section{Los dos paradigmas en la ciencia politica contemporánea: la mecánica clásica y el enfoque sistémico}

Iniciaremos señalando que entendemos por epistemología a la "rama de la filosofía que estudia la investigación científica y su producto, el conocimiento científico" (Bunge, 2006, p. 21). En ese sentido, la epistemología se pregunta sobre el método científico aplicado tanto en las ciencias naturales como en las ciencias sociales, es decir, en las ciencias fácticas (Bunge, 2007b). Para avanzar en esa dirección, aquí partimos de la idea de Mackenzie (1973), quien sostuvo que la ciencia política contemporánea es una disciplina que se expresa a través del "lenguaje de las variables, el rigor en el empleo de los modelos y los datos" (p. 460). En el inicio de la década de los setenta, W. J. Mackenzie (1973) hacía un balance sobre las tendencias epistemológicas y metodológicas de la ciencia política, destacando el nuevo "lenguaje de las variables”, herencia intelectual de Lazarsfeld (1973). Así, el esfuerzo de la ciencia política se concentraba en robustecer sus métodos de investigación que estaban fundamentados en los recientes avances en el campo de la estadística.

Aunado a lo anterior existía una intensa labor por establecer criterios metodológicos para construir bases de datos. Este nuevo paradigma en ciencia política se "inspiraba" en el modelo newtoniano de la ciencia fisicomatemática. El propio Mackenzie (1973) identifica a la escuela americana de ciencia política como una nueva corriente intelectual que se aparta de los estudios descriptivos de la historia, el derecho y la filosofía. Ahora, el desafío de la ciencia política era construir modelos formales de manera similar a la de las ciencias fisicomatemáticas. En primera instancia, las teorías del conductismo y, posteriormente, la elección racional le atribuía a la ciencia política dos características propias de la ciencia: a) la acumulación del conocimiento ${ }^{2}$ y b) la construcción de modelos generales (Adcock y Bevir, 2005).

Es así como la ciencia política se apartaba de la historia, pero ¿por qué se distanciaba la ciencia política de la historia en la tradición intelectual de la llamada escuela de ciencia política americana? Uno de los motivos que desarrollaremos a continuación es que la naciente ciencia política contemporánea se apoyaba epistemológicamente en el paradigma de la ciencia física newtoniana, es decir, en la concepción de la ciencia dentro de los parámetros de la mecánica clásica. Ahora el énfasis se pone en la identificación de variables para explicar el mundo político, no para describirlo dentro de los cánones tradicionales de la historia.

\footnotetext{
$1 \quad$ Traducción propia.

2 En su ensayo The Two-party System and Duverger's Law: An Essay on the History of Political Science el politólogo William H. Riker (1982) entiende por acumulación de conocimiento a "la forma más o menos verificable de proposiciones sobre el mundo natural" (p. 753).
} 
En 1942 Carl G. Hempel publica en The Journal of Philosophy el artículo The Function of General Laws in History. Este artículo despertó gran interés, tanto de historiadores como de politólogos, sobre la explicación científica aplicada en los procesos históricos. El trabajo de Hempel (1942) introducía la lógica de la explicación causal en la construcción del conocimiento histórico, pues de acuerdo con la argumentación de Hempel, el historiador podía superar el conocimiento meramente descriptivo para establecer tendencias en el análisis de los procesos históricos.

Para Hempel (1942) el quehacer científico del historiador es establecer e identificar eventos que se relacionen de forma causal, pues la "explicación causal es una explicación por leyes científicas" (p.37). El trabajo de Hempel (1942) derivó en el modelo nomológico de la explicación causal en la historia, 3 lo que hizo posible a los politólogos de la corriente del institucionalismo histórico, encontrar un terreno fértil, ya explorado analíticamente por Hempel, sobre la explicación causal de los procesos históricos. Asimismo, la obra de Hempel estableció las agendas de investigación para repensar la idea de explicación causal en las ciencias sociales en general y en la ciencia política en particular. No está de más mencionar que, dentro de esta misma corriente intelectual, uno de los libros más influyentes en el campo de la metodología de la ciencia política contemporánea es el de King, Keohane y Verba (1994), titulado Designing Social Inquiry: Scientific Inference in Qualitative Research, autores que postulan que la lógica de toda buena investigación es la inferencia, tanto en los estudios cuantitativos como en los cualitativos (McKeown, 1999).

En el análisis que hace O’Sullivan (2006) sobre el positivismo de Hempel en la filosofía de la historia de Goldstein, señala que para Hempel la explicación histórica consiste en presentar relaciones causales en el sentido de que "ciertos eventos de la clase $C$ son siempre seguidos por eventos de cierta clase E' (p. 206).4 Esta clase de relación causal es la estructura lógica de la explicación científica. De este modo, la historia como disciplina debe aspirar a descubrir leyes generales del tipo "las revoluciones son siempre seguidas por movimientos contrarrevolucionarios" (O’Sullivan, 2006, p. 206). La idea sobre la explicación causal desarrollada por Hempel está inspirada en la reflexión del filósofo David Hume sobre la conjunción constante entre dos eventos. Una explicación acabada sobre la relación causal es la que ofrece Gerring (2015):

Covariación (o correlación) se refiere a la relación mutua entre X e Y; y variación, al comportamiento de una sola variable. Estas palabras generalmente se usan de manera intercambiable. La palabra utilizada por Hume para esto era conjunción constante (constant conjunction) y otros también la han empleado de esta manera. Debo aclarar que, aunque el componente empírico de un argumento causal es covariacional por naturaleza, los argumentos causales exitosos dependen de más que solo covariación. Entre otras cosas, una explicación causal convincente debe identificar un mecanismo causal. (p.84)

El lenguaje de las variables a que se refiere Mackenzie (1973), se sustenta en la explicación causal de Hempel sobre los procesos históricos (Kitcher, 1989). En el fondo, la visión positivista se inserta en el paradigma de la mecánica clásica newtoniana, en donde pocas variables explican mucho, esto es, la visión parsimoniosa del mundo natural. Este paradigma de la mecánica clásica newtoniana, en sus premisas epistemológicas sobre la simplicidad de la naturaleza, es la que prevalece actualmente en la tradición intelectual predominante de la ciencia política contemporánea. Los modelos formales tienen así la capacidad de identificar las variables de observación, que expliquen el comportamiento de determinados fenómenos políticos. Como sostiene Ma (2007), la ciencia política se concibe como una "ciencia newtoniana" en la medida en que considera a la naturaleza como un sistema mecánico regido por leyes generales, que se expresan a través de las leyes formales de las matemáticas. Así, la "verdad científica establecida existe en un presente eterno. No hay necesidad de distinguir entre el pasado y el futuro" (Ma, 2007, p.60); en otras palabras, la "verdad científica" es ahistórica en la visión del

3 A este modelo nomológico de Hempel (1942) también se le conoce como la explicación científica de cobertura legal, compuesta por el explanans que contiene los enunciados de las condiciones antecedentes y los enunciados que representan las leyes generales que explican al fenómeno; y el explanandum que son los enunciados que describen el fenómeno. 
mundo de la mecánica clásica. En ese sentido, la visión positivista de Hempel sobre los procesos históricos fue importante en la medida en que insertó en la tradición de la historia y la ciencia política la explicación causal, cuyo instrumento poderoso estaba en la estadística. Sin embargo, esta concepción nos presentaría una idea rígida y estática de la realidad social, sin considerar los procesos históricos en su dinamismo y complejidad.

Es preciso destacar que gracias a Hempel se mantuvo la vieja tradición de las conexiones epistemológicas entre las ciencias naturales y las ciencias sociales. En una obra posterior Hempel y Oppenheim (1948) presentan una ampliación de los aspectos de la explicación científica, en donde insisten que para explicar los fenómenos que se presentan en el mundo de nuestra experiencia, habría que preguntarse el por qué, con el fin de dar con las causas que explican el fenómeno que observamos. A pesar de la fuerte influencia de Hempel, surge con nuevos bríos el enfoque sistémico en las ciencias sociales, que, sin abandonar la noción de explicación causal, retoma la complejidad multidimensional de los procesos históricos. Así, el enfoque sistémico fue abandonando gradualmente la limitada explicación mecanicista de los fenómenos políticos.

La complejidad de los procesos históricos y las dinámicas contingentes que conllevan los mismos, puso sobre la mesa de discusión la necesidad de explicar fenómenos políticos como resultado de largos procesos. Para realizar tal tarea era importante superar el paradigma mecanicista de la ciencia política actual, para dar comienzo a la discusión sistémica de los fenómenos políticos.

Si bien es cierto que la teoría de sistemas influyó en la ciencia política con la obra de Easton (1957), quien en su artículo An Approach to the Analysis of Political Systems había establecido como argumento central la existencia del sistema politico como una entidad autónoma, independiente de otros sistemas como la económica o psicológica. Con esta importante obra, el mundo político se comienza a concebir nuevamente como un fenómeno complejo. El enfoque sistémico, intenta observar tanto la parte como el todo, sin caer en los polos extremos del individualismo (la parte) y el holismo (el todo) (Bunge, 2007a). El propio enfoque sistémico de Bunge (2008), nos sirve aquí para ilustrar el modelo de sistema que planteamos (Ver Figura 1).

Escuriosoquelosinstitucionalistashistóricos hayan olvidado en su argumentación académica el trabajo de Easton (1957), estudioso que radicaliza la visión de la ciencia política al aplicar la teoría de sistemas en el análisis de la política. Para Ma (2007) la vertiente de la ciencia política contemporánea, que tiene elementos epistémicos sobre la "complejidad" del mundo social, es justamente el institucionalismo histórico. No es casual, que Pierson y Skocpol (2008), sostengan que el institucionalismo histórico es por derecho propio un enfoque de investigación en la ciencia política contemporánea, al igual que los enfoques del conductismo y el de elección racional. En palabras de Pierson y Skocpol (2008):

\begin{abstract}
$\mathrm{Al}$ igual que el personaje en la obra de Molière que habló en prosa durante toda su vida sin saberlo, los cientistas políticos contemporáneos están familiarizados con los ejemplos clásicos de la investigación del institucionalismo histórico sin por ello ser conscientes de que ejemplifican un género coherente, que cuenta con elementos que funcionan en forma similar al de los otros dos grandes enfoques de investigación de la ciencia política contemporánea, el conductismo basado en encuestas y los modelos de la elección racional. (p. 7)
\end{abstract}

Si bien Pierson y Skocpol (2008) encuentran en el institucionalismo histórico un enfoque propio de la ciencia política, describiendo de manera extraordinaria las contribuciones empíricas y teóricas en este campo de investigación, no se detienen a señalar la relevancia del enfoque sistémico que se encuentra detrás del institucionalismo histórico. Pues el institucionalismo histórico recoge en sus principales argumentos, que en ocasiones no están explícitas, las grandes contribuciones de los positivistas sobre la explicación causal de los procesos históricos, pero ahora enriquecido por la visión sistémica de la complejidad del mundo social. En ese sentido, una forma adecuada para analizar empíricamente los fenómenos políticos como procesos históricos dinámicos es a través de categorías como estrategias, trayectorias y coyunturas que a continuación describiremos en sus funciones explicativas.

\section{Las categorias de estrategias, trayectorias y coyunturas del institucionalismo histórico en la ciencia política contemporánea.}

$\mathrm{El}$ proyecto de investigación intelectual, en que actualmente dialogan la historia y la ciencia 
Nivel macrosocial (instituciones)

Apoyo o rebelión

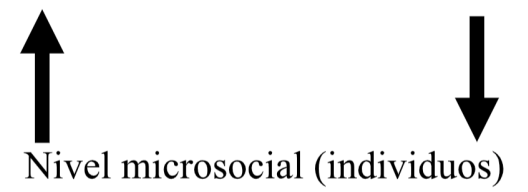

Asistencia o control

Fuente: Tomado de Bunge (2008), p. 20.

Figura 1. Modelo de sistema de acuerdo con Mario Bunge

política contemporánea de forma fructífera, es el institucionalismo histórico. El institucionalismo histórico recoge lo mejor de la herencia positivista sobre la explicación causal, introduciendo la idea de los "mecanismos causales" en la explicación de los procesos históricos; asimismo, su visión se ha enriquecido al introducir la complejidad propia del enfoque sistémico.

Es importante mencionar las corrientes intelectuales relevantes que han elaborado "teorías" generales de sistemas. Bunge (1999) señala que el problema de las teorías de sistemas es justamente eso: concebir la idea de sistema como una teoría. Más que una teoría de sistemas, Bunge (1999) explica que se trata más bien de un enfoque, que el investigador asume para articular áreas de conocimiento, superando así, la especialización en que se han encajonado las distintas disciplinas.

De acuerdo con lo anterior, identificamos, grosso modo, las siguientes corrientes sistémicas: i) La interpretación hermenéutica sobre los sistemas sociales de Luhmann (2006). El interés de Luhmann (2006) es realizar una interpretación de los significados del mundo social, sin detenerse en la epistemología de cómo opera en la realidad la investigación científica, algo que sí realiza Bunge (2007b) a través de su obra; ii) La teoría de sistemas sustentada en la formalización matemática y teoría de control de Bertoglio (1993), quien aborda su teoría de sistemas en modelos formales que provienen de la cibernética, teoría de juegos, teorías del control y la investigación de operaciones, pero no dedica su reflexión a las ciencias sociales como si lo hacen Bunge (1999) y Luhmann (2006); y iii) La propuesta de teoría de sistemas de Bertalanffy (1989) es más cercana a la obra de Bunge (2007b), por su inclinación a la epistemología de la investigación científica. A pesar de su inclinación a la epistemología de la investigación científica, Bertalanffy tiene una visión holística sobre la idea de sistema, que no permite capturar los procesos concretos en que se realiza la investigación empírica. Es por estas razones que nos inclinamos a pensar el enfoque sistémico desde la perspectiva del realismo crítico de Bunge (Hidalgo, 1982). Además, Bunge (2007a) ha participado en los debates sobre el proceso científico en las ciencias sociales, no a través de la hermenéutica (Luhmann, 2006), ni a través de una perspectiva exclusivamente tecnológica (Bertoglio, 1993), ni con un enfoque holístico (Bertalanffy,1989), sino más bien a través de las ideas que actualmente ocupan a la epistemología de la ciencia política contemporánea, concretamente sobre qué entendemos por explicación causal y mecanismos causales en las ciencias sociales (Gerring, 2015, 2010, 2005; Schmitter, 2009; Pierson y Skocpol, 2008; Przeworski, 2004; Mahoney, 2000, 2001b; King, Keohane y Verba, 1994; Riker, 1982).

En este contexto, analizamos en primer lugar la idea de mecanismo, para ello nos apoyamos nuevamente en Bunge (2008), quien ha dedicado penetrantes reflexiones sobre el tema. Bunge (2008) escribe:

En las ciencias y técnicas se habla sobre sistemas, y a veces también sobre emergencia y mecanismo. Por ejemplo, en biología humana se estudia el sistema cardiovascular; se dice que la circulación de la sangre es el mecanismo de oxigenación y limpieza de los tejidos; y que la arritmia o aun la fibrilación pueden sobrevenir o emerger como consecuencia de un estrés excesivo. Y los ingenieros saben que diseñan o reparan exclusivamente sistemas caracterizados por mecanismos que los mantienen en funcionamiento, y que deben permanecer alertas a la posible emergencia de nuevas cualidades, p. ej. una explosión causada por la combinación de elementos que tendrían que haberse mantenido separados. (p. 37) 
Los mecanismos son los factores que explican el funcionamiento de un sistema. En esta línea argumentativa, la explicación mecanísmica es un elemento central tanto en las ciencias naturales como en las ciencias sociales. Al igual que Hempel y Oppenheim (1948), Bunge (2008) destaca la importancia de contestar a la pregunta ¿por qué? para construir el conocimiento científico. En una acuciosa exposición nos dice Bunge (2008):

Si nos contenta la descripción no buscaremos mecanismos. Éstos importan sólo cuando se pretende entender, cuando se busca una explicación de lo que se ha descrito. Por ejemplo, se sabe que la globalización ha enriquecido a casi todas las naciones ricas, pero ha empobrecido a las demás. ¿Por qué?, o sea, ¿cuál ha sido el mecanismo que ha tenido este efecto? La respuesta más plausible es ésta: el libre comercio internacional facilita la cooperación global entre las naciones industrializadas al tiempo que refuerza la asimetría entre ellas y las agrarias. Por ejemplo, una nación productora de máquinas textiles y otra productora de tejidos pueden intercambiar ventajosamente sus productos. Pero una nación que sólo produzca algodón tendrá que venderlo a bajo precio, comprando en cambio a alto precio la maquinaria agrícola que usa para cultivarlo y cosecharlo, así como los vehículos que usa para transportarlo. En resumen, el libre comercio funciona ventajosamente entre iguales, pero no entre desiguales. (p. 44)

Llegamos al punto en el que es posible pensar la explicación mecanísmica dentro de los sistemas sociales, esto es, en el terreno complejo del mundo social. Asimismo, la explicación mecanísmica da cuenta de problemas formulados por un por qué. Un ejemplo que pone Bunge (2008) sobre los mecanismos causales en los fenómenos que estudia la ciencia política es el siguiente:

Con los mecanismos ocurre otro tanto: son tan reales como los sistemas que los poseen. Por ejemplo, la democracia política es un mecanismo de distribución del poder político: es una propiedad de ciertas sociedades, que distintas teorías conciben de maneras diferentes. Por ejemplo, Schumpeter piensa que la democracia consiste en un procedimiento para elegir autoridades, mientras que Dahl pone el acento sobre la dispersión del poder mediante la participación popular. (p. 43)

La concepción de los mecanismos es importante, porque se traduce en una herramienta analítica para estudiar los procesos históricos como sistemas, en donde se pueden identificar estrategias, trayectorias y coyunturas. Éstas categorías analíticas han enriquecido el lenguaje de la ciencia política para investigar en el tiempo a las instituciones políticas, las políticas públicas, el estado de bienestar, los movimientos sociales, entre otros fenómenos macrosociales (Pierson y Skocpol, 2008).

Estrategias. Uno de los temas notables en la ciencia política es la relación entre la agencia y la estructura (Peters y Pierre, 1998). La tensión entre agencia y estructura consiste en preguntarse cuál es el papel que desempeñan cada uno de ellos en el cambio institucional. La teoría de la elección racional en el ámbito de la ciencia política pone el énfasis en el actor racional (agencia), que al maximizar dentro del mercado político configura sus preferencias que le permitirán decidir. La tradición sociológica considera a la estructura como el factor relevante del cambio, un ejemplo son las organizaciones a través de sus rutinas y símbolos que configuran la conducta de los actores individuales. Hall y Taylor (1996) han señalado los rasgos distintivos con que trabajan los tres institucionalismos que ellos identifican: el institucionalismo histórico, el institucionalismo de la teoría de la elección racional y el institucionalismo sociológico (Ver Tabla 1).

Destacamos de la tipología de Hall y Taylor (1996), la idea de una relación sistémica en el institucionalismo histórico que incluye la parte (el individuo) y el todo (las instituciones), en una concepción dinámica del tiempo histórico. Así, el institucionalismo histórico nos muestra cómo la agencia se relaciona con la estructura. Además, que los actores individuales no se comportan de manera aislada, sino que configuran sus acciones con base en estrategias, analizando el comportamiento de los demás actores. Es por lo que el institucionalismo histórico tiene en las estrategias una herramienta analítica extraordinaria para estudiar las relaciones de poder entre los distintos actores.

Un ejemplo al respecto es el trabajo empírico de Mahoney (2001a), quien introduce la teoría de juegos para analizar las estrategias utilizadas por los actores políticos en el desarrollo político de los países centroamericanos de Guatemala, El Salvador, Costa Rica, Honduras y Nicaragua. En su trabajo nos explica los mecanismos causales que hicieron que en Guatemala y El Salvador surgiera un liberalismo radical, en Costa Rica un liberalismo reformista y en Honduras y Nicaragua 
que el liberalismo se abortara. En este orden de ideas, las estrategias como herramienta analítica del institucionalismo histórico le dan sentido a las relaciones de poder que se van configurando a través del tiempo. Esta concepción no está alejada del estudio de las estrategias del príncipe que describe Maquiavelo (1971) a través del estudio de la historia, ni de las estrategias de guerra confeccionadas por espartanos y atenienses en la Guerra del Peloponeso que nos narra el historiador Tucídides (1889).

Trayectoria (path dependence). Desde la antigüedad con Aristóteles (2016) se observa la importancia de los diseños constitucionales para conocer la arquitectura de los regímenes políticos. Con ello Aristóteles destaca la importancia de las instituciones para conocer la vida política de los pueblos. Las dos grandes revoluciones epistémicas de la ciencia política contemporánea, por un lado, el conductismo y por el otro la teoría de la elección racional, no contemplaron en su formulación inicial la importancia de las instituciones para explicar el mundo de la política. De acuerdo con Ma (2007), tanto el conductismo como la teoría de la elección racional, ambas tradiciones intelectuales ancladas en la visión newtoniana del mundo, se traducían a una concepción del mundo social de carácter ahistórico. Sin embargo, las instituciones empezaron a cobrar relevancia dentro de la ciencia política. De esta manera, las instituciones no se dan en el vacío, sino más bien en un contexto social histórico específico, marcado por los legados históricos y las contingencias de la historia (Ma, 2007).

Para los teóricos de la elección racional lo significativo del mundo de la política son los equilibrios y las preferencias de los individuos; por otro lado, para los institucionalistas históricos existen factores como el tiempo en donde se puede observar lo azaroso de la historia y la formación de las preferencias de los actores. Aquí, es importante mencionar, que los institucionalistas históricos, recurriendo a la vieja sabiduría grecorromana, consideran a la historia como eventos contingentes, anulando así la visión determinística de la historia en que se apoyaba la visión newtoniana del mundo.

Una de las categorías analíticas importantes del institucionalismo histórico, en donde la historia juega un papel crucial, es el de trayectoria (path dependence). Las trayectorias históricas tienen condiciones iniciales, cuyos resultados políticos desconocemos. Estas condiciones iniciales van

Tabla 1.

Características de los tres institucionalismos según Hall y Taylor

\section{Tipo de institucionalismo}

Institucionalismo histórico

\section{Caracteristicas}

Conceptualiza en términos más amplios la relación entre instituciones e individuos.

Se enfoca en las relaciones desiguales de poder en el desarrollo de las instituciones.

Contempla el desarrollo institucional a través de las categorias analiticas del "path dependence" (trayectorias) y de las "consecuencias no intencionadas".

Integra a las ideas para analizar los resultados políticos de las instituciones.

Institucionalismo de la teoría de la Toma elementos teóricos de la economía en el análisis del elección racional desarrollo institucional.

Pone el énfasis en los costos de transacción y los derechos de propiedad.

Institucionalismo sociológico Incorpora los sistemas simbólicos, mapas cognitivos y las normas morales que configuran los "marcos de significado" de las instituciones.

Fuente: Hall y Taylor (1996). 
generando eventos en el tiempo de acuerdo con las decisiones que los actores vayan tomando, de tal forma que lo actores van eligiendo alternativas con el paso del tiempo (Pierson, 2000). El análisis de la trayectoria explica los mecanismos causales por los cuales se dan determinados resultados políticos: por qué los actores eligieron la alternativa A, pero no la B. En el mundo social de los teóricos del institucionalismo histórico nada está determinado; es más, puede darse la situación que un evento aparentementeinsignificantemarqueuna trayectoria que configure a las instituciones políticas de un contexto social determinado. Przeworski (2004) nos brinda, de manera elocuente, una reflexión sobre las condiciones iniciales infinitamente pequeñas, para ser más precisos, no observables:

Efectivamente, sabemos que algunas situaciones estratégicas pueden generar cualquiera de entre diversos resultados, equilibrios múltiples. Incluso si la historia es dependiente del camino recorrido, puede exhibir una dependencia sensible a condiciones iniciales infinitamente pequeñas, esto es, no observables. Aquí tenemos un ejemplo ilustrativo. El heredero de una familia destacada, rica, conservadora y católica de Costa Rica fue enviado a estudiar al extranjero en 1920, tal y como su trasfondo familiar dictaba. Lo único que ocurrió fue que, en vez de ir a París, aterrizó en la Universidad de Louvain, en un momento en el cual era un semillero de ideología socialcristiana. Impregnado por estas ideas, volvió a su país, se convirtió en su presidente, se alió con el Partido Comunista, legalizó los sindicatos e introdujo una extensa legislación social. Alarmados por su alianza con los comunistas, los Estados Unidos ingeniaron un golpe de Estado, dirigido por un socialdemócrata anti-comunista. A partir de entonces, en Costa Rica se alternaron social-cristianos y socialdemócratas, todos respetuosos con la democracia y todos comprometidos con la igualdad. Entonces, si esta historia es cierta, una perturbación minúscula tuvo grandes y duraderas consecuencias. ${ }^{5}$ (párr. 60)

Como agudamente observa Przeworski (2004) una "perturbación minúscula" marcó el resultado político de todo un país. La explicación histórica plausible de Przeworski es analíticamente poderosa porque ilustra lo azaroso e imperceptible de la historia, lo complejo que suelen ser los eventos históricos en la constitución de las instituciones políticas. Los resultados sociales de estas "perturbaciones minúsculas", o bien como dice Pierson (2000) de "eventos contingentes" producen consecuencias de un amplio "rango de resultados posibles”. Esta visión del tiempo histórico corresponde más al paradigma sistémico de un mundo complejo, que al paradigma determinístico del sistema newtoniano de la ciencia. En otra formulación de Pierson y Skocpol (2008), sobre la forma de observar estos pequeños eventos contingentes de las trayectorias desde el ángulo de la causalidad, es la siguiente:

Quizás más relevante, una apreciación de la prevalencia del path dependence nos obliga a prestar atención a las dimensiones temporales de los procesos políticos. Destaca el rol de lo que Arthur Stinchcombe ha denominado "causalidad histórica' en la que las dinámicas desatadas por un evento o proceso en un punto en el tiempo se reproducen, incluso en ausencia de la recurrencia del evento o proceso original. (p. 13)

El pasaje anterior destaca por dos razones: a) por concebir a la política como un "proceso" en el tiempo, una secuencia de eventos que pueden ser analizados en las trayectorias, para conocer las dinámicas propias de las disputas por el poder y las fuerzas que emergen en dichas disputas por el poder; b) la conexión epistemológica entre la explicación científica que propone Hempel (1942), pero ahora a través de los mecanismos causales dentro de un sistema. Nuestra pretensión en el presente trabajo es llamar la atención sobre la explicación causal en los trabajos clásicos de Hempel (1942). No obstante, existe tal pluralidad de tipologías de causas que Gerring (2005) se ha dado a la tarea de construir un marco unificado para las ciencias sociales sobre la explicación causal.

Coyunturas. Una categoría, que viene a enriquecer el lenguaje analítico del institucionalismo histórico como un cuerpo teórico coherente, es el de coyunturas. Las coyunturas son "entendidas como efectos de interacción entre distintas secuencias causales que se unen en determinados puntos del tiempo" (Pierson y Skocpol, 2008, p. 15). Si bien es cierto que el politólogo no realizará la tarea del historiador, sí debe tener las competencias

5 Es importante hacer notar que el marco teórico de Adam Przeworski es la teoría de la elección racional y la teoría de juegos. Sin embargo, su crítica epistemológica al nuevo institucionalismo aporta elementos valiosos para reflexionar en torno al institucionalismo histórico. 
suficientes para identificar las coyunturas en los procesos históricos.

Como nos recuerdan Collier y Collier (1991)

la categoría de coyuntura crítica fue elaborada por Seymour Martin Lipset y Stein Rokkan para explicar las transiciones históricas ${ }^{6}$. De este modo, la literatura que analiza, por ejemplo, la transición democrática en América Latina pone el énfasis en las coyunturas críticas, que abren paso a distintas dinámicas democráticas a través de los países de América Latina. Sobre el modelo de las coyunturas críticas para estudiar los procesos de democratización en América Latina, escribe Munck (2010):

Un cuarto cuerpo de literatura sobre regimenes y democracia en América Latina se ha basado en un modelo de coyuntura crítica y ha presentado una familia de argumentos que se basan en este modelo. Una característica distintiva de estos modelos es que explican desarrollos políticos en términos de los legados de eventos que se produjeron muchas décadas en el pasado en lugar de postular causas y efectos que están cerca en el tiempo, como es más habitual en las teorías expuestas anteriormente. Otra característica clave es que los modelos de coyuntura crítica, aunque invocando variables económicas y sociales, suelen incorporar variables políticas como factores explicativos centrales y por lo tanto proponen teorías que sistemáticamente reconocen la autonomía de la política. Así, aunque la literatura sobre coyunturas críticas es muy diversa, introduce una ruptura significativa con respecto a las tres líneas de investigación discutidas anteriormente y ofrece una alternativa a las teorías societales a través de su énfasis en explicaciones históricas y políticas. (p. 20)

En resumen, las coyunturas como modelo de explicación son productivas empíricamente. El institucionalismo histórico, al incorporar las estrategias, las trayectorias y las coyunturas, supone un mundo social complejo en donde los sistemas están interconectados. El diálogo entre la historia y la ciencia política contemporánea, permite revalorar la importancia del tiempo. Como escribe el físico Prigogine (2006): "Hoy, bien o mal, todos están de acuerdo sobre la importancia de la evolución en cosmología, en las partículas elementales, en biología, en las ciencias humanas; todos están de acuerdo en la importancia del tiempo” (p. 24).

\section{Conclusiones}

Las preguntas de investigación que nos planteamos al inicio de este capítulo fueron las siguientes: ¿Cuáles son las conexiones epistemológicas entre la historia y la ciencia política? ¿Cuál es el papel analítico que juegan categorías como estrategias, trayectorias y coyunturas en los estudios de procesos históricos que actualmente usa el enfoque sistémico en el institucionalismo histórico? En torno a estas preguntas indagamos a través del diálogo entre la historia y la ciencia política contemporánea.

Respecto a la primera pregunta, nuestra respuesta tentativa es que los enunciados epistemológicos que subyacen actualmente en la ciencia política tienen que ver con la concepción de ciencia que se tiene. En este sentido, los dos paradigmas predominantes son la visión mecanicista (newtoniana) y el enfoque sistémico. La concepción newtoniana de la ciencia está hecha de simplicidad en la explicación y de modelos parsimoniosos que explican el mundo político. Este modelo de ciencia tiene grandes aportaciones, como señalamos la idea de explicación causal que plantea Hempel; si bien esto fue un gran avance tanto dentro de la historia, como de la ciencia política, una nueva generación de investigadores de la ciencia política, en aras de buscar nuevos paradigmas, retomaron el enfoque sistémico. El enfoque sistémico fue asumido dentro de las tradiciones intelectuales de la ciencia política por el institucionalismo histórico. Esta tradición de pensamiento sostiene el diálogo entre la historia y la ciencia política, como sucedió con los clásicos como Tucídides (1889) y Maquiavelo (1971). El institucionalismo histórico es posible debido a la concepción sistémica del mundo social, recuperando así, un elemento importante de la modernidad, que es justamente ver el mundo como un sistema, en toda su complejidad.

Nuestra segunda pregunta de investigación sostiene una respuesta tentativa a partir del aparato teórico y empírico que se ha venido produciendo en la ciencia política, a través de la tradición intelectual del institucionalismo histórico. En el momento en que la ciencia política toma en serio el tiempo tuvo que recurrir a la historia, construyendo las categorías de estrategias, trayectorias y coyunturas, que como expusimos, han sido poderosas en la explicación de procesos políticos. Estudiar los procesos políticos a través del tiempo, encaminó a

6 En este trabajo tomamos de manera indistinta el concepto de "coyuntura" y "coyuntura crítica". 
los politólogos a tomar a la historia como la ciencia que proporciona la evidencia empírica para observar el comportamiento de los actores en largos períodos de tiempo y en sus interconexiones sistémicas.

Finalmente, nuestra investigación es un avance que pretende analizar los supuestos epistemológicos en que se sustenta la ciencia política en diálogo con la historia. Estos supuestos no están explícitos en los trabajos empíricos del institucionalismo histórico, pero creemos importante hacerlos explícitos para observar con claridad la concepción de ciencia que se intenta establecer en la ciencia política contemporánea. Además, nuestra apuesta, es por un diálogo transdisciplinario, con un enfoque sistémico, que enriquezca la investigación tanto teórica, como metodológica de la ciencia política actual que practicamos en América Latina en aras de construir instituciones políticas que demandan nuestras sociedades complejas.

\section{Referencias}

Adcock, R. \& Bevir, M. (2005). The History of Political Science. Political Studies Review, 3(3), 1-16.

Aristóteles (2016). La política. D.F., México: UNAM.

Bertalanffy, L. von (1989). Teoría general de los sistemas. Fundamentos, desarrollo, aplicaciones. D.F., México: Fondo de Cultura Económica.

Bertoglio, O. J. (1993). Introducción a la teoría general de sistemas. D.F., México: Limusa.

Bunge, M. (1999). Sistemas sociales y filosofía. Buenos Aires, Argentina: Sudamericana.

Bunge, M. (2006). Epistemología. D.F., México: Siglo XXI.

Bunge, M. (2007a). Buscar la filosofia en las ciencias sociales. D.F., México: Siglo XXI.

Bunge, M. (2007b). La investigación cientifica. D.F., México: Siglo XXI.

Bunge, M. (2008). Dos enfoques: sectorial y sistémico. En M. Bunge. Filosofia y sociedad (pp. 9-23). D.F., México: Siglo XXI.

Collier, R. B. \& Collier, D. (1991). Shaping the Political Arena: Critical Junctures, the Labor Movement and Regime Dynamics in Latin America. Princeton, N. J.: Princeton University Press.

Colomer, J. M. (2007). Instituciones Politicas. Barcelona, España: Ariel.

Easton, D. (1957). An Approach to the Analysis of Political Systems. World Politics, 9(3), 383-400.

Gerring, J. (2005). Causation: A Unified Framework for the Social Sciences. Journal of Theoretical Politics, 17(2),163-198.

Gerring, J. (2010). Causal Mechanisms: Yes, but... Comparative Political Studies, 43(11), 1499-
1526 .

Gerring, J. (2015). ¿Qué es un estudio de caso y para qué sirve? En R. Castiglioni y C. Fuentes S. (Eds.), Política comparada sobre América Latina: teorías, métodos y tópicos (pp. 79-116). Santiago de Chile: Ediciones Universidad Diego Portales.

Hall, P. A. \& Taylor, R. C. R. (1996). Political Science and the Three New Institutionalisms. Political Studies, 44, 936-957.

Hempel, C. G. (1942). The Function of General Laws in History. The Journal of Philosophy, 39(2), 3548.

Hempel, C. G. y Oppenheim, P. (1948). Studies in the Logic of Explanation. Philosophy of Science, 15(2), 135-175.

Hidalgo, A. (1982). Entrevista con Mario Bunge. El Basilisco, 14, 64-73.

Hobbes, T. (2005). Leviatán. O la material, forma y poder de una república eclesiástica y civil. D.F., México: Fondo de Cultura Económica.

King, G., Keohane, R. O. y Verba, S. (1994). Designing Social Inquiry. Scientific inference in Qualitative Research. Princeton, N. J.: Princeton University Press.

Kitcher, P. (1989). Explanatory Unification and the Causal Structures of the World. En Kitcher y Salmon (eds.), Scientific Explanation (pp. 7191). Minneapolis, MN: University of Minnesota Press.

Lazarsfeld, P. F. (1973). La sociología. En J. Piaget, P. F. Lazarsfeld, W.J.M. Mackenzie et al. Tendencias de la investigación en las ciencias sociales (pp. 283-438). Madrid, España: Alianza.

Luhmann, N. (2006). La sociedad de la sociedad. D.F., México: Herder-Universidad Iberoamericana.

Ma, S. Y. (2007). Political Science at the Edge of Chaos? The Paradigmatic Implications of Historical Institutionalism. International Political Science Review, 28(19), 57-78.

Mackenzie, W. J. W. (1973). La ciencia política. En J. Piaget, P. F. Lazarsfeld, W.J.M. Mackenzie et al. Tendencias de la investigación en las ciencias sociales (pp. 439-528). Madrid, España: Alianza.

Mahoney, J. (2000). Path Dependence in Historical Sociology. Theory and Society, 29(4), 507-548.

Mahoney, J. (2001a). Radical, Reformist and Aborted Liberalism: Origins of National Regimes in Central America. Journal of Latin American Studies, 33(2), 221-256.

Mahoney, J. (2001b). Beyond Correlational Analysis: Recent Innovations in Theory and Method. Sociological Forum, 15(3), 575-593.

Maquiavelo, N. (1971). El Príncipe. En N. Maquiavelo 
Obras Politicas (pp. 302-359). La Habana, Cuba: Editorial de Ciencias Sociales, Instituto Cubano del Libro.

McKeown, T. J. (1999). Case Studies and the Statiscal Worldview: Review of King, Keohane, and Verba's Designing Social Inquiry: Scientific Inference in Qualitative Research. International Organization, 53(1),161-190.

Munck, G. L. (2010). Los orígenes y la durabilidad de la democracia en América Latina: avances y retos de una agenda de investigación. Revista de Ciencia Política, 30(3), 573-597.

O’Sullivan, L. (2006). Leon Goldstein and the Epistemology of Historical Knowing. History and Theory, 45(2), 204-228.

Peters, B. G. y Pierre, J. (1998). Institutions and Time: Problems of Conceptualization and Explanation. Journal of Public Administration Research and Theory, 8(4), 565-583.

Pierson, P. (2000). Increasing Returns, Path Dependence, and the Study of Politics. American Political Science Review, 94(2), 251-267.

Pierson, P. y Skocpol, T. (2008). El institucionalismo histórico en la ciencia política contemporánea. Revista Uruguaya de Ciencia Política, 17(1),7-38.
Prigogine, I. (2006). El nacimiento del tiempo. Buenos Aires, Argentina: Tusquets.

Przeworski, A. (2004). La Última Instancia: ¿Son las Instituciones la Causa Primaria del Desarrollo Económico? Recuperado del Departamento de Política de la Universidad de New York: http://politics.as.nyu.edu/docs/IO/2800/ archives_spanish.pdf.

Riker, W. H. (1982). The Two-Party System and Duverger's Law: An Essay on the History of Political Science. American Political Science Review, 76(4), 753-766.

Schmitter, P. C. (2009). The nature and future of comparative politics. European Political Science Review, 1(1), 33-61.

Tucídides (1889). Historia de la Guerra del Peloponeso. Madrid, España: Librería de la Viuda de Hernando y C. ${ }^{\text {a }}$

Weinstein, D. (2012). Herbert Spencer. Recuperado de Stanford Encyclopedia of Philosophy: http:// plato.stanford.edu/entries/spencer/. 